\title{
Diagnostic value of dynamic contrast-enhanced magnetic resonance imaging in rectal cancer and its correlation with tumor differentiation
}

\author{
FU SHEN, JIANPING LU, LUGUANG CHEN, ZHEN WANG and YUKUN CHEN \\ Department of Radiology, Changhai Hospital, Shanghai 200433, P.R. China
}

Received September 8, 2015; Accepted January 22, 2016

DOI: $10.3892 / \operatorname{mco} .2016 .762$

\begin{abstract}
Dynamic contrast-enhanced magnetic resonance imaging (DCE-MRI) is a novel imaging modality that can be used to reflect the microcirculation, although its value in diagnosing rectal cancer is unknown. The present study aimed to explore the clinical application of DCE-MRI in the preoperative diagnosis of rectal cancer, and its correlation with tumor differentiation. To achieve this, 40 pathologically confirmed patients with rectal cancer and 15 controls were scanned using DCE-MRI. The Tofts model was applied to obtain the perfusion parameters, including the plasma to extravascular volume transfer (Ktrans), the extravascular to plasma volume transfer (Kep), the extravascular fluid volume (Ve) and the initial area under the enhancement curve (iAUC). Receiver-operating characteristic (ROC) curves were plotted to determine the diagnostic value. The results demonstrated that the time-signal intensity curve of the rectal cancer lesion exhibited an outflow pattern. The Ktrans, Kep, Ve, and iAUC values were higher in the cancer patients compared with controls $(\mathrm{P}<0.05)$. The intraclass correlation coefficients of Ktrans, Kep, Ve and iAUC, as measured by two independent radiologists, were 0.991, 0.988, 0.972 and 0.984 , respectively (all $\mathrm{P}<0.001$ ), indicating a good consistency. The areas under the ROC curves for Ktrans and iAUC were both $>0.9$, resulting in a sensitivity and specificity of $100 \%$ and $93.3 \%$ for Ktrans, and of $92.5 \%$, and $93.3 \%$ or $100 \%$, for iAUC, respectively. In the 40 rectal cancer cases, there was a moderate correlation between Ktrans and iAUC, and pathological differentiation $(0.3<r<0.8$, all $\mathrm{P}<0.05)$. In conclusion, Ktrans and iAUC were associated with the presence of rectal cancer and differentiation, and therefore may provide novel insights into the preoperative diagnosis of rectal cancer.
\end{abstract}

Correspondence to: Dr Jianping Lu, Department of Radiology, Changhai Hospital, 168 Chang Hai Road, Shanghai 200433, P.R. China

E-mail: cjr.lujianping@vip.163.com

Key words: rectal cancer, dynamic contrast-enhanced magnetic resonance imaging, differentiation, diagnosis, tumor differentiation

\section{Introduction}

Rectal cancers affect the rectum, and are generally adenocarcinomas (1). Nearly $70 \%$ of patients are $>65$ years old, and rectal cancer occurs rarely in patients who are under 40 years of age; in addition, men are predominantly affected (2). Probable risk factors include age, male gender, colon polyps, a history of colorectal cancer, a history of inflammatory bowel disease, hereditary syndromes, lifestyle factors (diet, alcohol, obesity, sedentary lifestyle and smoking), and a history of diabetes mellitus (1). Rectal cancer is usually managed using a combination of surgery, chemotherapy, targeted therapy and radiation therapy (3). The five-year survival rates following surgical resection are $85-95 \%$ for stage I, $60-80 \%$ for stage II, and $30-60 \%$ for stage III cancer. With the aging of the Chinese society and a Westernization of the diet, the incidence of rectal cancer in China is increasing (4). Therefore, finding novel means to efficiently detect and diagnose rectal cancer are required.

Dynamic contrast-enhanced magnetic resonance imaging (DCE-MRI) is a relatively novel imaging modality that demonstrates the capillary blood flows $(5,6)$. In DCE-MRI, the distribution of the contrast agent is repeatedly evaluated, allowing the evaluation of the tumor microcirculation in vivo and enabling the malignancy or benignancy of the tumor to be quantitatively distinguished $(6,7)$. A number of previous studies have shown the diagnostic value of DCE-MRI for prostate cancer (8-10), for the evaluation of pancreatic cancer (11), and for evaluating the efficacy of cancer drugs (12-14). Only one previous study has used DCE-MRI to evaluate the tumor drug response in colorectal cancer (15), and, at present, no published studies have been concerned with the diagnostic value of DCE-MRI for rectal cancer.

Therefore, the two-fold aim of the present study was to use DCE-MRI to compare the tumor perfusion parameters with postoperative pathology results in order to elucidate the characteristics of the DCE-MRI parameters in rectal cancer, and to explore the application of DCE-MRI in the preoperative diagnosis of rectal cancer.

\section{Materials and methods}

Subjects. Patients with a suspected rectal lesion (blood in stool, changes of bowel habits, and/or suspicious masses identified 
by finger examination or colonoscopy) were recruited at the Anorectal Surgery Department, Changhai Hospital Affiliated to the Second Military Medical University (Shanghai, China), between December 2013 and February 2015. All subjects underwent DCE-MRI and postoperative pathology. Inclusion criteria were: i) A single lesion on conventional imagery; and ii) no chemotherapy or radiotherapy was administered prior to DCE-MRI. Patients were excluded if: i) DCE-MRI images were of poor quality; ii) images did not include the whole lesion; iii) artifacts affected measurements; or iv) lesions were too small to be accurately measured.

Finally, 55 patients were included in the study, 40 of whom had been diagnosed with pathology-confirmed rectal cancer, whereas the remaining 15 subjects were included as controls. These 15 controls included two cases of chronic inflammation, one case of rectum hemorrhoids, one case of isolated hamartoma, six cases of tubular adenoma, three cases of tubular villous adenoma and two healthy controls.

The present study was approved by the Committee on Ethics of Biomedical Research, Second Military Medical University (Shanghai, China). All subjects signed a written informed consent.

MRI scanning. MRI was performed using a Skyra 3.0 T MRI scanner (Siemens AG, Erlangen, Germany) with a pelvic phased array coil. All subjects had fasted for $4 \mathrm{~h}$ prior to scanning. Routine pelvic MRI and DCE-MRI sequences were used for scanning. The routine sequences included sagittal T2 weighted imaging (T2WI) with fat suppression, axial T1WI, T2WI, diffusion (D)WI, and coronal T2WI. DCE-MRI was used to scan the same axial layers as T2WI with the following scanning parameters: Repetition time (TR) $3.33 \mathrm{msec}$, echo time (TE) $1.23 \mathrm{msec}$, flip angle $9^{\circ}$, field of view (FOV) $36 \mathrm{~cm}$, matrix 125x192, layer thickness $3 \mathrm{~mm}$ and 30 layers; parallel acquisition by CAIPIRINHA and acceleration factor $\mathrm{R}=2$. Each scan lasted for $5 \mathrm{sec}$, and 75 scans were performed with free respiration. Following the initial scan, the contrast agent, gadolinium-diethylenetriaminepentacetate (Beijing Hokuriku Pharmaceutical Co., Ltd., China; volume of $15-20 \mathrm{ml}, 0.2 \mathrm{mmol} / \mathrm{kg}$ ) was injected intravenously at $3 \mathrm{ml} / \mathrm{sec}$ using high-pressure syringes. Subsequently, $20 \mathrm{ml}$ saline solution of $0.90 \%(\mathrm{w} / \mathrm{v}) \mathrm{NaCl}$ was injected at the same rate.

Measurements and data analysis. All data obtained from the DCE sequences were sent to the Tissue 4D post-processing workstation (Siemens AG) for post-processing. Parameter calculations were performed using the Tofts pharmacokinetic model $(16,17)$. On the DCE images, an area resembling a circle, including all rectal and surrounding mesangial regions, was selected to generate DCE-MRI pseudo-color images. The images were examined in a blind manner by two radiologists specializing in MRI, with 5 years of experience. By comparing with the T2WI images, the radiologists selected the plane sections where the lesions were relatively large, based on the morphology, sites, infiltration depth, enhancement patterns and invasion into surrounding regions to manually place regions of interest (ROI), with areas no less than $1 \mathrm{~cm}^{2}$. The perfusion parameters $(16,17)$ were measured from three plane sections, and the average was used for analysis: i) volume transfer constant from the plasma compartment to the extravascular extracellular space (Ktrans); ii) rate constant for transfer between the extravascular extracellular space and the blood compartment (Kep); iii) volume of extravascular extracellular space per unit volume of tissue (Ve); and iv) the initial area under the enhancement curve (iAUC). Time-signal intensity curves were plotted.

Patients with rectal cancer were divided into three groups, according to the tumor differentiation based on the postoperative pathology reports evaluated in a blind manner by two pathologists with 5 years of experience: i) highly differentiated; ii) moderately differentiated; and iii) poorly differentiated (18).

Statistical analysis. SPSS version 16.0 software (SPSS, Inc., Chicago, IL, USA) was used for the statistical analysis. Data are expressed as the means \pm standard deviation. Due to the heterogeneity of variance in the parameters between patients with rectal cancer and controls, the Mann-Whitney U test was used to assess the differences between the two groups. Intraclass correlation coefficients (ICCs) were used to estimate the consistency of the results from the two radiologists. To determine the diagnostic power of Ktrans, Kep, Ve, and iAUC, the receiver operator characteristics (ROC) curve method was used, and areas under the curve AUCs were calculated. Optimal cut-off values were selected to maximize specificity and sensitivity. The $\mathrm{H}$ test was used to compare DCE-MRI parameters among different degrees of differentiation. The correlations between parameters and differentiation of rectal cancer were analyzed using the Spearman's rank correlation test. $\mathrm{P}<0.05$ was considered to indicate a statistically significant difference.

\section{Results}

Characteristics of the patients. Among the 40 patients with rectal cancer, there were 20 males and 20 females, aged 57 \pm 9 (range 25-68) years. Among the 15 controls, there were eight males and seven females, aged 56 \pm 7 (range 44-70) years. No significant differences in gender or age were identified between the two groups.

DCE-MRI manifestation of rectal cancer. All 40 cases of rectal cancer had a single lesion, of which 26 cases exhibited space-occupying masses, 10 revealing an irregular thickening of the local intestinal wall, and four cases showing abnormal local nodular signals. All lesions demonstrated equal or lower T1WI signals, equal or higher T2WI signals, equal or higher fat-suppression sequence signals, and high DWI signals. Significantly enhanced lesions were capable of being viewed on DCE-MRI. The Ktrans, Kep, Ve, and iAUC values in rectal cancer lesions were all significantly higher compared with the controls (all $\mathrm{P}<0.05)$ (Table I). The pseudo-color images of each parameter are shown in Figs. 1 and 2. The time-signal intensity curves showed an outflow pattern: The signal peaked shortly following the injection of the contrast agent, and subsequently quickly decayed by $>10 \%$ compared with the signal intensity in the mid- and late-stage of the enhancement after the contrast agent was injected (Fig. 1G). On the other hand, in the controls, the time-signal intensity 
Table I. DCE-MRI parameters.

\begin{tabular}{lcccccc}
\hline Group & $\mathrm{n}$ & Radiologist & Ktrans $\left(\mathrm{min}^{-1}\right)$ & Kep $\left(\mathrm{min}^{-1}\right)$ & Ve $(\%)$ & iAUC \\
\hline Rectal cancer & 40 & 1 & $0.267 \pm 0.071$ & $0.615 \pm 0.212$ & $0.489 \pm 0.101$ & $37.177 \pm 8.845$ \\
& & 2 & $0.257 \pm 0.070$ & $0.605 \pm 0.202$ & $0.474 \pm 0.081$ & $35.800 \pm 8.093$ \\
Controls & 15 & 1 & $0.118 \pm 0.032$ & $0.427 \pm 0.163$ & $0.361 \pm 0.166$ & $16.052 \pm 5.828$ \\
& & 2 & $0.121 \pm 0.027$ & $0.411 \pm 0.160$ & $0.367 \pm 0.148$ & $17.328 \pm 4.428$ \\
P-value $^{\mathrm{a}}$ & & 1 & $<0.001$ & 0.005 & 0.003 & $<0.001$ \\
& & 2 & $<0.001$ & 0.002 & 0.008 & $<0.001$ \\
\hline
\end{tabular}

Data are shown as the mean \pm standard deviation. ${ }^{a}$ The Mann-Whitney U test was used to compare DCE-MRI parameters among patients with rectal cancer and controls. DCE-MRI, dynamic contrast-enhanced magnetic resonance imaging; Ktrans, volume transfer constant from the plasma compartment to the extravascular extracellular space; Kep, rate constant for transfer between extravascular extracellular space and the blood compartment; Ve, volume of extravascular extracellular space per unit volume of tissue; iAUC, initial area under enhancement curve.
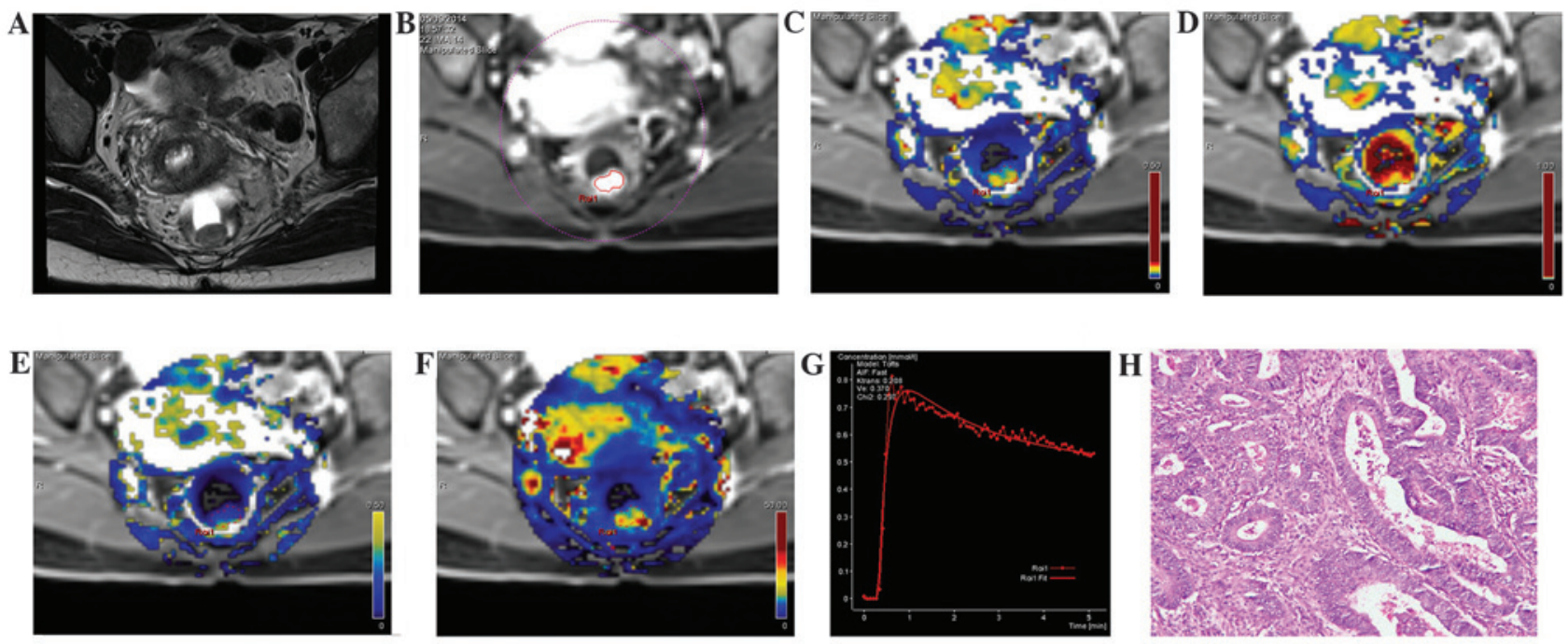

Figure 1. MRI images from the same patient with rectal cancer, a female aged 48 years with blood in the stool for 6 months. (A) A T2WI cross section, revealing the local occupation of the rear intestinal wall. (B) DCE-MRI scanning image showing a marked enhancement of the rear intestinal wall, which was slightly higher than the surrounding regions. (C) DCE-MRI pseudo-color images of Ktrans. The yellowish-green area indicates the local occupation of the intestinal wall. Ktrans of the lesion area was 0.208 , which was higher compared with the surrounding intestinal wall tissue (blue). (D) DCE-MRI pseudo-color images of Kep. The red and yellow areas indicate the lesion area, where Kep was 0.588. (E) DCE-MRI pseudo-color images of Ve. The yellowish-green area indicates the lesion area, where Ve was 0.370. (F) DCE-MRI pseudo-color images of iAUC. The red and yellow areas indicate the lesion area, where iAUC was 37.009, which was higher compared with the surrounding intestinal wall tissue (blue). (G) The DCE-MRI time-signal intensity curve of the patient, where the dashed line indicates the connection between data points and the solid line indicates the fitted curve, reveals an outflow pattern. (H) Results of the postoperative pathology test (hematoxylin and eosin staining, x200). The tumor tissue showed a papillary and mesh-like alignment. Tumor cells were cubic and of a column-like shape, with big, atypical and deeply stained nuclei, which infiltrated into the superficial muscle layer of the intestinal wall. The patient was diagnosed with moderately differentiated rectal adenocarcinoma. DCE-MRI, dynamic contrast-enhanced magnetic resonance imaging; Ktrans, volume transfer constant from the plasma compartment to the extravascular extracellular space; Kep, rate constant for transfer between extravascular extracellular space and the blood compartment; Ve, volume of extravascular extracellular space per unit volume of tissue; iAUC, initial area under enhancement curve.

curves revealed a comparatively smaller elevation of signal intensity at the early stage, and a plateau at the later stage (Fig. 2G).

The ICCs between the two independent measurements were $0.991,0.988,0.928$ and 0.984 , for Ktrans, Kep, Ve and iAUC, respectively (all $\mathrm{P}<0.001$ ) (Table II), which suggested a good consistency of the measurements, and indicated the reliability and reproducibility of the DCE-MRI method.

ROC curve analysis of DCE-MRI parameters. ROC curve analysis was performed based on the independent measurement of the DCE-MRI parameters (Ktrans, Kep, Ve and
Table II. Consistency of the results from the two radiologists.

\begin{tabular}{lrrrr}
\hline Variable & Ktrans & Kep & Ve & iAUC \\
\hline ICC $^{\text {a }}$ & 0.991 & 0.988 & 0.928 & 0.984 \\
P-value & $<0.001$ & $<0.001$ & $<0.001$ & $<0.001$ \\
\hline
\end{tabular}

aCC, intraclass correlation coefficient. Ktrans, volume transfer constant from the plasma compartment to the extravascular extracellular space; Kep, rate constant for transfer between extravascular extracellular space and the blood compartment; Ve, volume of extravascular extracellular space per unit volume of tissue; iAUC, initial area under enhancement curve. 

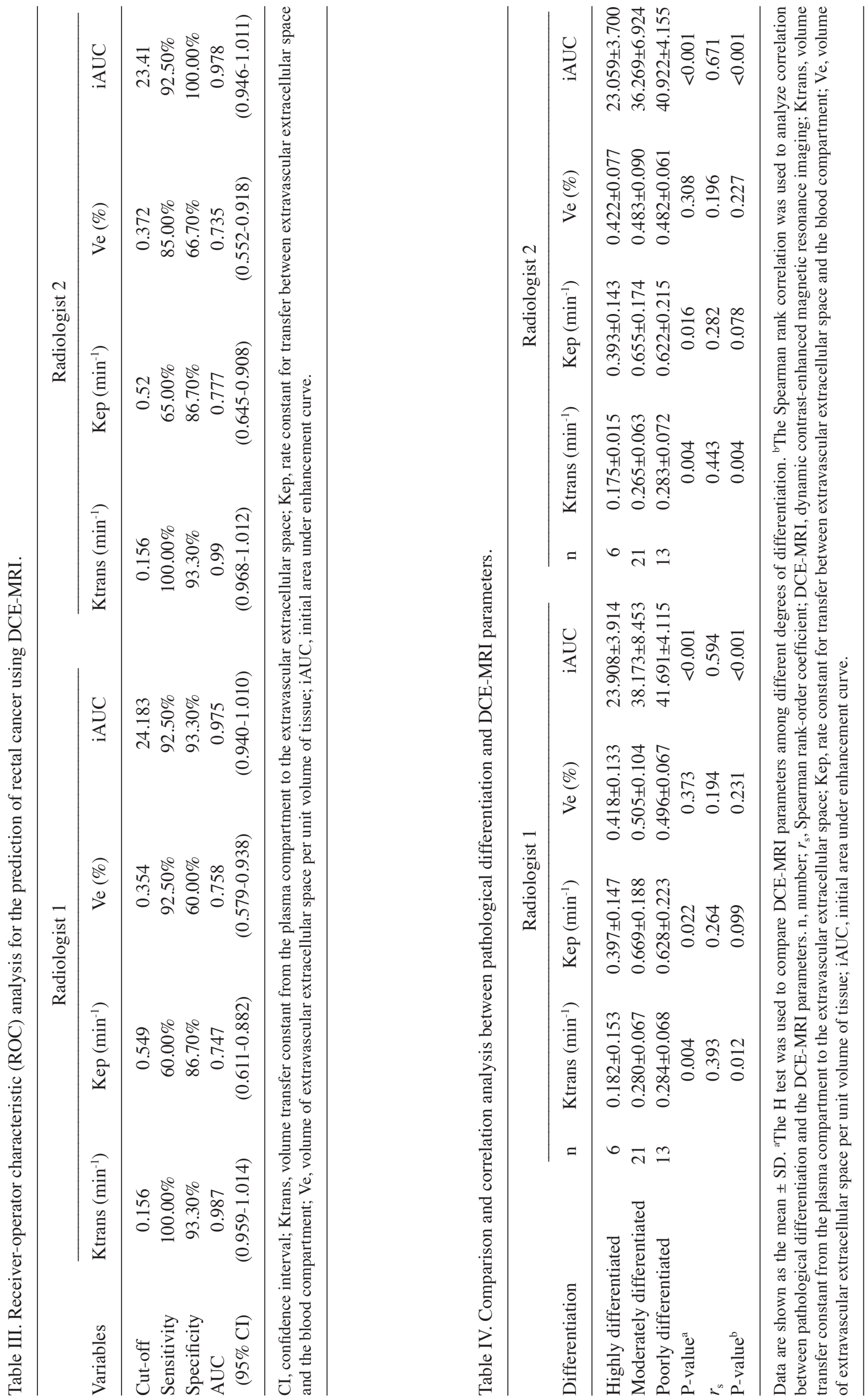

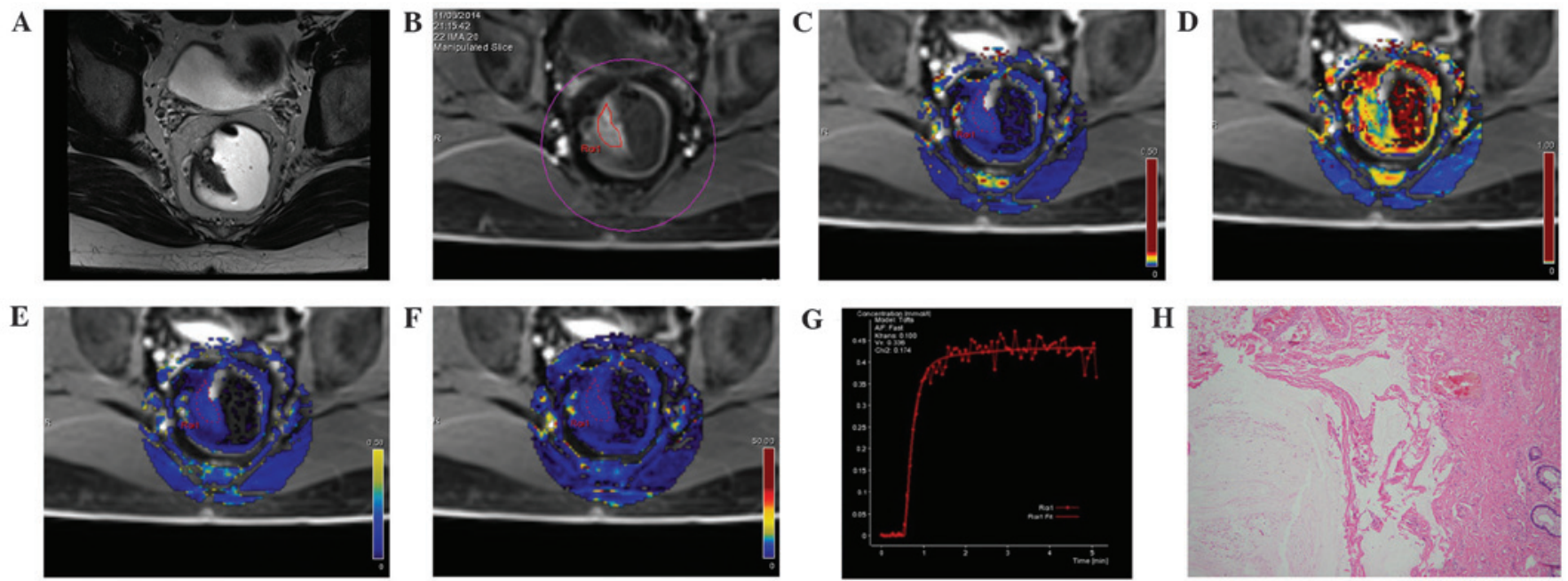

Figure 2. MRI images from the same patient in the control group: A male, aged 35 years, with a feeling of incomplete defecation for one year, occasional anal pain, and slight blood in the stool. Swollen masses were identified by finger examination. (A) A T2WI cross-section showing abnormal occupation signals on the right side of the intestinal wall. (B) DCE-MRI scanning images showing similar enhancement of the space occupation of the selected intestinal wall compared with the surrounding regions. (C) DCE-MRI pseudo-color images of Ktrans. The blue color was evenly distributed along the intestine, and the Ktrans of the ROI was 0.100. (D) DCE-MRI pseudo-color images of Kep. The lesion area was blue, where Kep was 0.428. (E) DCE-MRI pseudo-color images of Ve. The lesion area was blue, where Ve was 0.336. (F) DCE-MRI pseudo-color images of iAUC. The lesion area was blue, where iAUC was 18.834, which was comparable with the surrounding intestinal wall tissue. (G) The DCE-MRI time-signal intensity curve of the control revealed a pattern of a rapid rise, followed by a plateau. (H) Results of the postoperative pathology test (hematoxylin and eosin staining, x100). Cysts were formed in the intestinal submucosa, filled with mucus. Mucus overflow formed a mucus paste outside several of the cysts. No atypical gland structures or cells were observed surrounding the cysts. Fibrosis, chronic inflammatory cell infiltration, and vascular dilatation and congestion were seen in the submucosa. The patient in the control group was diagnosed with colitis cystica profunda. DCE-MRI, dynamic contrast-enhanced magnetic resonance imaging; Ktrans, volume transfer constant from the plasma compartment to the extravascular extracellular space; Kep, rate constant for transfer between extravascular extracellular space and the blood compartment; Ve, volume of extravascular extracellular space per unit volume of tissue; iAUC, initial area under enhancement curve.
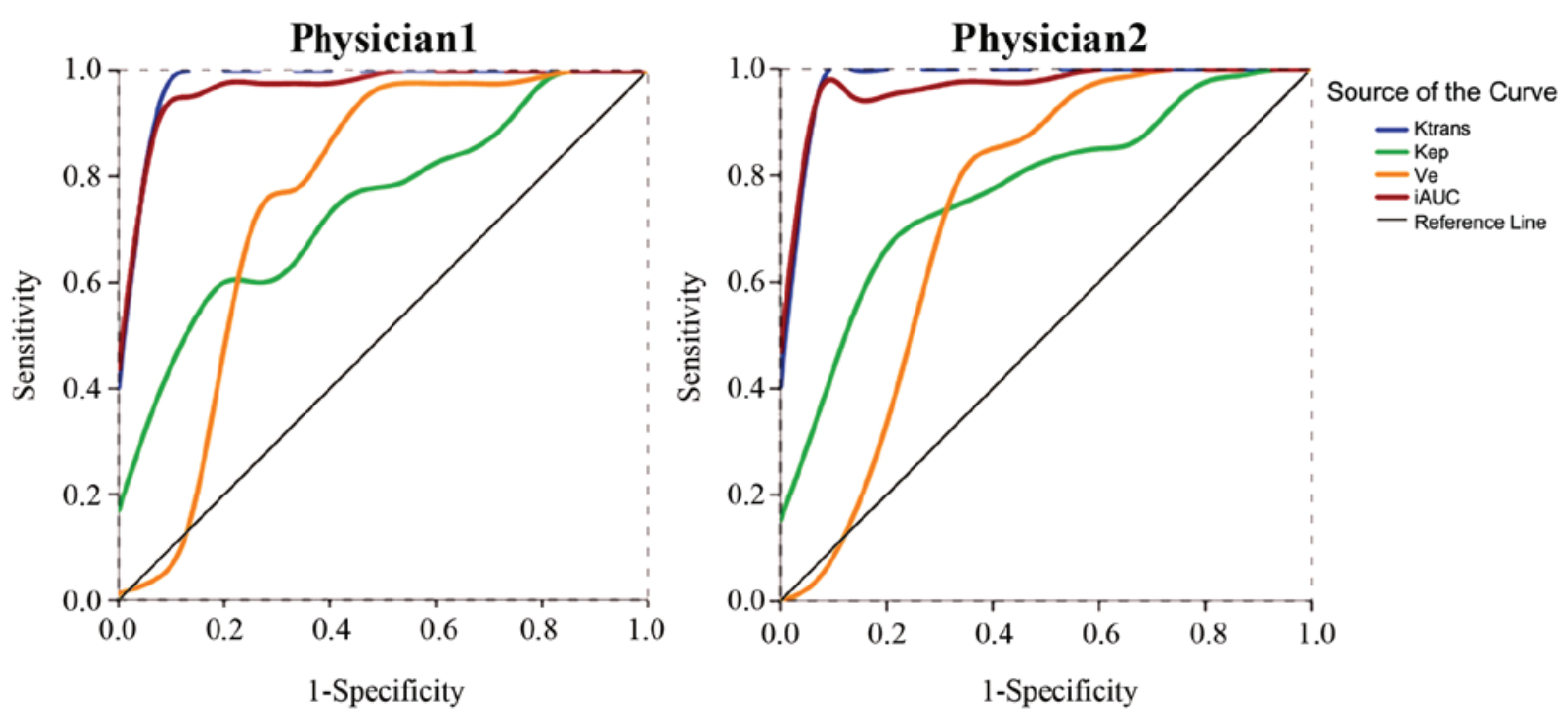

Figure 3. Receiver operating characteristic curves of the dynamic contrast-enhanced magnetic resonance imaging parameters measured independently by two radiologists. The AUCs of Ktrans (blue) and iAUC (red) were both $>0.9$, with great consistency in measurement. Ktrans, volume transfer constant from the plasma compartment to the extravascular extracellular space; Kep, rate constant for transfer between extravascular extracellular space and the blood compartment; Ve, volume of extravascular extracellular space per unit volume of tissue; (i)AUC, (initial) area under enhancement curve.

iAUC) from the two radiologists. The areas under the ROC curves were $0.987,0.747,0.758$ and 0.975 from radiologist 1 , and $0.990,0.777,0.735$ and 0.978 from radiologist 2 , with great consistency in measurement (Fig. 3). Using a $0.156 \mathrm{~min}^{-1}$ cut-off value for Ktrans, the two radiologists determined a sensitivity of $100.0 \%$ and a specificity of $93.3 \%$. Using a cut-off value of 24.183 or 23.410 for iAUC, the sensitivity was $92.5 \%$ for both analyses, and the specificity was $93.3 \%$ or $100.0 \%$, respectively. A detailed analysis of the results are shown in Table III.

Correlation between DCE-MRI parameters and pathological differentiation. The pathology reports revealed that, out of the 40 cases of rectal cancer, there were 13 poorly 
differentiated cases of cancer, 21 moderately differentiated cancers, and 6 well differentiated cancers. According to radiologist 1 , there were significant differences in Ktrans (poorly: $0.284 \pm 0.068$ and moderately: $0.280 \pm 0.067$ vs. well; $0.182 \pm 0.153 \mathrm{~min}^{-1}, \mathrm{P}=0.004$ ), Kep (poorly: $0.628 \pm 0.223$ and moderately: $0.669 \pm 0.188$ vs. well; $0.397 \pm 0.147 \mathrm{~min}^{-1}$, $\mathrm{P}=0.022$ ), and iAUC (poorly: $41.69 \pm 4.12$ and moderately: $38.17 \pm 8.45$ vs. well; $23.91 \pm 3.91, \mathrm{P}<0.001)$ among three groups, but not for $\mathrm{Ve}(\mathrm{P}=0.373)$. Out of the four parameters, Ktrans $(r=0.393, \mathrm{P}=0.012)$ and iAUC $(r=0.594, \mathrm{P}<0.001)$ were correlated with differentiation, whereas Kep and Ve were not significantly correlated with pathological differentiation (Table IV). Similar results were obtained by radiologist 2 .

\section{Discussion}

Currently, the diagnosis of rectal cancer relies on traditional imaging. DCE-MRI is a relatively novel MRI technology that combines morphology and changes in hemodynamics, and can quantitatively evaluate tumor differentiation in a more accurate way (19). Therefore, the aim of the present study was to explore the clinical application of DCE-MRI in the preoperative diagnosis of rectal cancer and its correlation with tumor differentiation. The results showed that Ktrans, Kep, Ve and iAUC were higher in cancer patients compared with controls. The time-signal intensity curve of the rectal cancer lesion revealed an outflow pattern. The areas under the ROC curves for Ktrans and iAUC were both $>0.9$, resulting in a sensitivity and specificity of $100 \%$ and $93.3 \%$ for Ktrans, and of $92.5 \%$, and $93.3 \%$ or $100 \%$, for iAUC, respectively. The ICCs of Ktrans, Kep, Ve, and iAUC measured by the two independent radiologists were $0.991,0.988,0.972$ and 0.984 , respectively, indicating a good consistency. In the 40 rectal cancer cases, there was a moderate correlation between Ktrans and iAUC and pathological differentiation.

Higher blood vessel density resulting from tumor proliferation, and its synergistic effect with abnormal molecular regulation within the tumor cells, lead to abnormal angiogenesis, which is represented by leakage, twisted morphology, vascular wall expansion and crosslinking (20). This abnormal morphology results in a loose connection or loss of pericytes that nourish the endothelial cells, and huge gaps between the endothelium and basement membrane, and between the basement membrane and pericytes, leading to enlargement of the gap between vascular endothelial cells and attenuation of the maturity of blood vessels, which consequently leads to high permeability and vulnerability of newborn tumor blood vessels (21).

The most commonly used DCE-MRI parameter that reflects vascular permeability is Ktrans (the volume transfer constant) $(16,22)$. Ktrans represents the rate at which the contrast agent transfers from the blood to the interstitium, which indicates the tumor microcirculation and the surface infiltration area. In contrast, Kep, the reverse rate constant, reflects the rate at which the contrast agent transfers from the extravascular extracellular space back to the blood. Ve is the fractional extravascular leakage volume, which predominantly reflects the percentage of contrast agent in the extravascular extracellular space (16). In addition, the semi-quantitative parameter, iAUC, is associated with tumor blood influx, perfu- sion and interstitium, and represents the general tumor blood flow, overall perfusion and tumor interstitial space index (16).

The present study revealed that Ktrans, Kep, Ve, and iAUC were higher in patients with rectal cancer compared with controls, indicating that massive angiogenesis and abnormal vasculature enhanced the influx of contrast agent, whereas the incomplete development of vascular endothelial cells and high vascular permeability led to increased leakage of contrast agent. However, the arteriovenous connection was observed to cause a perfusion shortcut (22), which supports the results of the present study.

Both ROC curves from the two radiologists demonstrated a large AUC ( $>0.9)$ for Ktrans and iAUC, suggesting that these two parameters had high sensitivity and specificity compared with the other two parameters. In addition, the present study also revealed that Ktrans and iAUC were correlated with the pathological differentiation of rectal cancer $(0.3<r<0.8)$, which increased when the tumor was less differentiated. This may be partially explained by the altered vascular permeability by tumor angiogenesis, as well as the fact that poorly-differentiated tumors had more cells at metaphase, which required more nutritive elements and higher blood perfusion, and therefore a higher Ktrans. Furthermore, poorly differentiated tumors exhibited a greater heterogeneity of cell morphology and histology, higher cell density and smaller interstitium.

Neither Kep nor Ve were significantly correlated with tumor differentiation. It may be surmised that Kep and Ve were associated with the composition of extravascular, extracellular space and, despite the high local vascular permeability, the composition of the extravascular, extracellular space did not markedly differ among lesions with different differentiation statuses. Further investigation is necessary to explore the clinical relevance of these two parameters.

The results of the present study suggest that DCE-MRI parameters can be used to distinguish malignant lesions from benign ones. These results are in agreement with previous studies performed with other types of solid tumors, including orbital (23), breast $(24,25)$, head and neck (26) and pancreatic tumors (11). A previous study in colorectal cancer is consistent with the present study (27). Another previous study also demonstrated that DCE-MRI parameters may be correlated with tumor differentiation in rectal cancer (28).

The present study is not without limitations. First, solid tumors are highly heterogeneous, and intestinal lesions often have an irregular shape. Occasionally, they cannot be distinguished from the surrounding adipose tissue due to inflammation and blood vessel invasion. If the ROI is placed at the boundary of the tumor, the results may vary, and multiple measurements are required to obtain the average. Secondly, the patients were grouped according to the state of differentiation, and the sample size was small for the well-differentiated group. Finally, DEC-MRI is a relatively novel imaging modality, and it is not yet standardized. Future studies should include more patients in order to validate the DCE-MRI measurements for rectal cancer diagnosis.

In conclusion, the present study has confirmed that DCE-MRI parameters may reflect the difference in microcirculation of rectal cancer, and Ktrans and iAUC were correlated with rectal cancer differentiation, which may provide an effective preoperative diagnosis modality for rectal cancer. 


\section{References}

1. Cunningham D, Atkin W, Lenz HJ, Lynch HT, Minsky B, Nordlinger B and Starling N: Colorectal cancer. Lancet 375: 1030-1047, 2010.

2. Labianca R, Nordlinger B, Beretta GD, Mosconi S, Mandalà M, Cervantes A and Arnold D; ESMO Guidelines Working Group: Early colon cancer: ESMO clinical practice guidelines for diagnosis, treatment and follow-up. Ann Oncol 24 (Suppl 6): vi64-vi72, 2013.

3. Clinical practice guidelines in oncology (NCCN Guidelines): Colon cancer. Version 3.2015. National comprehensive cancer network, Fort Washington, 2015.

4. Chen WQ, Zeng HM, Zheng RS, Zhang SW and He J: Cancer incidence and mortality in china, 2007. Chin J Cancer Res 24: 1-8, 2012.

5. Hylton N: Dynamic contrast-enhanced magnetic resonance imaging as an imaging biomarker. J Clin Oncol 24: 3293-3298, 2006.

6. Jackson A, O'Connor JP, Parker GJ and Jayson GC: Imaging tumor vascular heterogeneity and angiogenesis using dynamic contrast-enhanced magnetic resonance imaging. Clin Cancer Res 13: 3449-3459, 2007.

7. Cha S: Perfusion MR imaging: Basic principles and clinical applications. Magn Reson Imaging Clin N Am 11: 403-413, 2003.

8. Villers A, Puech P, Mouton D, Leroy X, Ballereau C and Lemaitre L: Dynamic contrast enhanced, pelvic phased array magnetic resonance imaging of localized prostate cancer for predicting tumor volume: Correlation with radical prostatectomy findings. J Urol 176: 2432-2437, 2006.

9. Haider MA, Chung P, Sweet J, Toi A, Jhaveri K, Ménard C, Warde P, Trachtenberg J, Lockwood G and Milosevic M: Dynamic contrast-enhanced magnetic resonance imaging for localization of recurrent prostate cancer after external beam radiotherapy. Int J Radiat Oncol Biol Phys 70: 425-430, 2008.

10. Puech P, Potiron E, Lemaitre L, Leroy X, Haber GP, Crouzet S, Kamoi K and Villers A: Dynamic contrast-enhanced-magnetic resonance imaging evaluation of intraprostatic prostate cancer: Correlation with radical prostatectomy specimens. Urology 74: 1094-1099, 2009.

11. Liu K, Xie P, Peng W and Zhou Z: Dynamic contrast-enhanced magnetic resonance imaging for pancreatic ductal adenocarcinoma at 3.0-T magnetic resonance: Correlation with histopathology. J Comput Assist Tomogr 39: 13-18, 2015.

12. Hahn OM, Yang C, Medved M, Karczmar G, Kistner E, Karrison T, Manchen E, Mitchell M, Ratain MJ and Stadler WM: Dynamic contrast-enhanced magnetic resonance imaging pharmacodynamic biomarker study of sorafenib in metastatic renal carcinoma. J Clin Oncol 26: 4572-4578, 2008.

13. Liu G, Rugo HS, Wilding G, McShane TM, Evelhoch JL, $\mathrm{Ng} \mathrm{C}$, Jackson E, Kelcz F, Yeh BM, Lee FT Jr, et al: Dynamic contrast-enhanced magnetic resonance imaging as a pharmacodynamic measure of response after acute dosing of AG-013736, an oral angiogenesis inhibitor, in patients with advanced solid tumors: Results from a phase I study. J Clin Oncol 23: 5464-5473, 2005.

14. Barrett T, Davidson SR, Wilson BC, Weersink RA, Trachtenberg J and Haider MA: Dynamic contrast enhanced MRI as a predictor of vascular-targeted photodynamic focal ablation therapy outcome in prostate cancer post-failed external beam radiation therapy. Can Urol Assoc J 8: E708-E714, 2014.

15. Morgan B, Thomas AL, Drevs J, Hennig J, Buchert M, Jivan A, Horsfield MA, Mross K, Ball HA, Lee L, et al: Dynamic contrast-enhanced magnetic resonance imaging as a biomarker for the pharmacological response of PTK787/ZK 222584, an inhibitor of the vascular endothelial growth factor receptor tyrosine kinases, in patients with advanced colorectal cancer and liver metastases: Results from two phase I studies. J Clin Oncol 21: 3955-3964, 2003.
16. Tofts PS, Brix G, Buckley DL, Evelhoch JL, Henderson E, Knopp MV, Larsson HB, Lee TY, Mayr NA, Parker GJ, et al: Estimating kinetic parameters from dynamic contrast-enhanced T(1)-weighted MRI of a diffusable tracer: Standardized quantities and symbols. J Magn Reson Imaging 10: 223-232, 1999.

17. Franiel T, Hamm B and Hricak H: Dynamic contrast-enhanced magnetic resonance imaging and pharmacokinetic models in prostate cancer. Eur Radiol 21: 616-626, 2011.

18. Bosman FT: World Health Organization: International agency for research on cancer. WHO classification of tumours of the digestive system. International Agency for Research on Cancer, Lyon, 2010.

19. Vriens D, van Laarhoven HW, van Asten JJ, Krabbe PF, Visser EP, Heerschap A, Punt CJ, de Geus-Oei LF and Oyen WJ: Chemotherapy response monitoring of colorectal liver metastases by dynamic Gd-DTPA-enhanced MRI perfusion parameters and 18F-FDG PET metabolic rate. J Nucl Med 50: 1777-1784, 2009.

20. Furuya M and Yonemitsu Y: Cancer neovascularization and proinflammatory microenvironments. Curr Cancer Drug Targets 8: 253-265, 2008.

21. Jain RK: Normalization of tumor vasculature: An emerging concept in antiangiogenic therapy. Science 307: 58-62, 2005.

22. Teifke A, Behr O, Schmidt M, Victor A, Vomweg TW, Thelen M and Lehr HA: Dynamic MR imaging of breast lesions: Correlation with microvessel distribution pattern and histologic characteristics of prognosis. Radiology 239: 351-360, 2006.

23. Yuan Y, Kuai XP, Chen XS and Tao XF: Assessment of dynamic contrast-enhanced magnetic resonance imaging in the differentiation of malignant from benign orbital masses. Eur J Radiol 82: 1506-1511, 2013.

24. Nadrljanski M, Maksimović R, Plešinac-Karapandžić V, Nikitović M, Marković-Vasiljković B and Milošević Z: Positive enhancement integral values in dynamic contrast enhanced magnetic resonance imaging of breast carcinoma: Ductal carcinoma in situ vs. invasive ductal carcinoma. Eur J Radiol 83: 1363-1367, 2014

25. Wang S, Delproposto Z, Wang H, Ding X, Ji C, Wang B and $\mathrm{Xu} \mathrm{M}$ : Differentiation of breast cancer from fibroadenoma with dual-echo dynamic contrast-enhanced MRI. PLoS One 8: e67731, 2013

26. Ishiyama M, Richards T, Parvathaneni U and Anzai Y: Dynamic contrast-enhanced magnetic resonance imaging in Head and Neck Cancer: Differentiation of new H\&N cancer, recurrent disease and benign post-treatment changes. Clin Imaging 39: 566-570, 2015

27. Tuncbilek N, Karakas HM and Altaner S: Dynamic MRI in indirect estimation of microvessel density, histologic grade and prognosis in colorectal adenocarcinomas. Abdom Imaging 29: 166-172, 2004

28. Hong HS, Kim SH, Park HJ, Park MS, Kim KW, Kim WH, Kim NK, Lee JM and Cho HJ: Correlations of dynamic contrast-enhanced magnetic resonance imaging with morphologic, angiogenic and molecular prognostic factors in rectal cancer. Yonsei Med J 54: 123-130, 2013. 